\title{
Investigation and Research on Patients' Preferences and Values in The Development of Clinical Practice Guidelines of Traditional Chinese Medicine for Primary Headache
}

\author{
JinKe Huang, YangYang Wang, RunSheng Xie and Hui Li* \\ Research Office of Standardization, Second Affiliated Hospital, Engineering and Technology Research Center of Standardization of \\ Traditional Chinese Medicine, China \\ *Corresponding author: Hui Li, Guangdong Provincial Hospital of Traditional Chinese Medicine, Guangzhou City, Guangdong, China
}

\section{ARTICLE INFO}

Received: 幽 February 23, 2019

Published: 慧 March 13, 2019

Citation: Limin Liu, Wanlin Xu, Shengwen Liu, Shukun Shen, Francia Fang, Wenjun Yang, Jiang Li. PLAG1: Diagnostic Utility in atypical Pleomorphic Adenoma. Biomed J Sci \& Tech Res 15(5)2019. BJSTR. MS.ID.002773.

Keywords: Primary Headache; Guidelines; Preferences; Values

\section{ABSTRACT}

Background: According to the latest evidence-based guidelines for clinical practice formulate principles, the Clinical Practice Guidelines of Traditional Chinese Medicine for Primary Headache, which are based on the GRADE system, should take appropriate account of patient's preferences and values.

Objective: To study the preferences and values of patients with primary headache and to provide research evidence to the development of clinical practice guidelines of traditional Chinese medicine for primary headache.

Methods: A cross-sectional survey was conducted. Besides, visual simulation score (VAS) was used to measure patients' preferences and values in outcome indicators, the direction of overall treatment measures, syndrome differentiation and treatment of TCM, and specific treatment measures for primary headache.

Results: There are differences in outcome indicators, the direction of overall treatment measures, and the tendency of migraine and tension-type headache intervention measures among patients $(\mathrm{P}<0.01)$. There was no significant difference in the tendency of syndrome differentiation and treatment of TCM or treatment of cluster headache $(P>0.01)$.

Conclusion: The outcome indicators of patients' tendency were in turn: prevention and reduction of recurrence, less adverse reactions, the convenience of receiving treatment and recovery, rapid pain relief, whether it was within the coverage of medical insurance, and treatment cost. In terms of the direction of overall treatment measures, non-drug therapy is the first choice, among which acupuncture and massage have a higher tendency than other therapies. Drug therapy is a secondary choice, which in turn tends to traditional Chinese medicine, traditional Chinese medicine decoction, western medicine. In the treatment of migraine, the tendency of acupuncture, collaterals puncture and bloodletting was higher than that of tou tonging-capsule. In the treatment of tension-type headache, the tendency of massage is higher than that of tou tonging-capsule. There is no special preference for syndrome differentiation of TCM and cluster headache treatment. Evidence-based medicine (ebm) points out that the best clinical decisions should organically combine patients' preferences and values, clinician skills and experience, and clinical research evidence together. With the development of evidence-based medicine and systematic review and its impact on clinical practice guidelines, the American Academy of Medical Sciences updated its definition of clinical practice guidelines for the first time in two decades in 2011, emphasizing that a trustworthy guideline must take patients' preferences and values into account.

Through systematic search and screening, a total of 23 guidelines related to TCM diagnosis and treatment of headache were obtained, but none of which considered of 
patients' preferences and values. Clinical Diagnosis and Treatment Guide for TCM Internal Medicine (Headache) 2017 (registration number: IPGRP-2015CN005), which is recognized as China's first clinical practice guideline of traditional Chinese medicine included in the national clinical diagnosis and treatment guideline database $(\mathrm{Ngc})$ of the United States, its formulation method is in strict accordance with the WHO guideline formulation principles [1-3]. The need to systematically investigate and study the preferences and values of headache patients to provide research evidence to assist in the formulation of the guideline is emphasized, which is one of the important features in it.

\section{Participants and Methods}

\section{Participants}

Patients who have suffered from headache and have had at least one headache attack in the past year.

\section{Research Content}

Basic Information About the Disease: The questionnaire provides references for diagnosis and treatment of headache. The diagnosis includes headache types, clinical manifestation and differentiation. The name of specific Chinese herbal decoction and non-Chinese herbal decoction, efficacy, adverse reactions, quality of evidence, average cost and other information of specific treatment measures are provided in the treatment field. Evidence was collected through literature and systematic rating, and then the quality of evidence was obtained by GRADE recommendation. The average cost was estimated by combining the single treatment cost with that required during treatment period.

Basic Information About the Survey Content: The investigation contents include five aspects: patients 'basic situation, the outcome indicator of interest, the direction of the general therapeutic measures, treatment method to headache by differentiation of TCM and the specific therapeutic measures of primary headache. The basic information of patients includes six items: gender, current residence, age, ethnicity, education degree and headache in the past year. The outcome indicators mainly focus on seven items, including cure, prevention and reduction of recurrence, adverse reactions, convenience of treatment, rapid pain relief, cost and medical insurance. The direction of general therapeutic measures includes drug therapy and non-drug therapy. The TCM syndrome differentiation and treatment of headache includes nine items: wind-cold headache, wind-heat headache, rheumatism headache, hyperactivity of liver Yang, disturbance of phlegm and turbidity, stasis of blood and collaterals, deficiency of qi and blood, deficiency of liver and kidney Yin, stagnation of liver and qi. Specific treatments for primary headaches include three items such as migraine, tension headaches and cluster headaches.

\section{Methods}

From May to August 2017, cross-sectional survey method was adopted to provide two-dimensional code of online questionnaire for headache patients in outpatient department through questionnaire website (www.sojump.com). After scanning the code, the patients referred to the basic information of the disease provided by the questionnaire and filled in the questionnaire according to their own feelings and experience.

The basic information of the patients was collected in the forms of single choice, multiple choice and blank filling. There were 6 items in total. Through visual simulation score (VAS), the tendency of outcome indicators concerned by patients, the orientation of overall treatment measures, the tendency of TCM syndrome differentiation for headache treatment, and the tendency of specific treatment measures for primary headache were measured, with a total of 21 items. In which scoring (1-9) of the outcome indicator, 1 point means thar it is completely inconsequential, and 9 points means that itis very important. Scoring of the direction of overall treatment measures, TCM syndrome differentiation for headache, and specific treatment measures for primary headache (1-9), 1 points for not using, and 9 points for definitely using.

\section{Statistical Methods}

Use Excel software for data entry and SPSS20.0 software for data analysis. Covariance analysis was also employed for it using of $\mathrm{P}<0.01$ for differences, which was statistically significant.

\section{Results}

\section{Status of The Questionnaire}

There were total 217 participants in the survey. The age was from 17 to 82, including 72 men, 145 women. 79 of them received college or secondary education and below and 138 with bachelor degree and above.

\section{Outcome Indicators}

The influence of gender, age and educational level factors was controlled by covariance analysis method, and the results shows that there was a significant difference in the tendency of patients to outcome index $(\mathrm{P}<0.01)$, as shown in Table 1 . a represents the correction mean after the control covariance, the rest of the table is the same. 
Table 1: Tendency of outcome indicators.

\begin{tabular}{|c|c|c|c|c|c|}
\hline \multirow{2}{*}{ Serial Number } & \multirow{2}{*}{ Items } & \multirow{2}{*}{ Average } & \multicolumn{2}{|c|}{$95 \%$ Confidence Interval } & \multirow{2}{*}{$\boldsymbol{P}$} \\
\hline & & & Lower & Ceiling & \\
\hline 1 & Prevention and reduction of recurrence & $8.288^{a}$ & 7.854 & 8.721 & $P<0.01$ \\
\hline 2 & Less adverse reactions & $8.175^{\mathrm{a}}$ & 7.742 & 8.608 & \\
\hline 3 & Cure & $8.150^{\mathrm{a}}$ & 7.717 & 8.583 & \\
\hline 4 & Ease of access to treatment & $7.587^{\mathrm{a}}$ & 7.154 & 8.021 & \\
\hline 5 & Quick pain relief & $7.163^{\mathrm{a}}$ & 6.729 & 7.596 & \\
\hline 6 & Whether Within the coverage of Medicare & $5.988^{\mathrm{a}}$ & 5.554 & 6.421 & \\
\hline 7 & Cost of treatment & $5.838^{a}$ & 5.404 & 6.271 & \\
\hline
\end{tabular}

\section{Direction of The Overall Treatment Measures}

After eliminating the influence factors by covariance analysis, such as gender, age and educational level,the result shows that there is a difference in the direction tendency of the patients to the overall treatment measures $(\mathrm{P}<0.01)$, as shown in Table 2. 2.4
TCM syndrome Differentiation and treatment After eliminating the influence factors by covariance analysis, such as gender, age and educational level,the result shows that there is no significant difference in the syndromes and decoction tendency of TCM syndrome differentiation in patients with headache $(\mathrm{P}>0.01)$, as shown in Table 3.

Table 2: Direction of overall treatment measures.

\begin{tabular}{|c|c|c|c|c|c|c|c|}
\hline \multirow{2}{*}{ Serial Number } & \multirow{2}{*}{\multicolumn{2}{|c|}{ Items }} & \multirow{2}{*}{$\begin{array}{c}\text { Average } \\
\text { Lower }\end{array}$} & \multicolumn{2}{|c|}{ 95\% Confidence Interval } & \multirow{2}{*}{\multicolumn{2}{|c|}{$\boldsymbol{P}$}} \\
\hline & & & & Ceiling & & & \\
\hline \multirow{3}{*}{1} & \multirow{3}{*}{ Non-drug therapy } & Massage & $7.275^{\mathrm{a}}$ & 6.755 & 7.795 & \multirow{3}{*}{$P<0.01$} & \multirow{6}{*}{$P<0.01$} \\
\hline & & Acupuncture & $6.562 \mathrm{a}$ & 6.043 & 7.082 & & \\
\hline & & Other & $5.325^{\mathrm{a}}$ & 4.805 & 5.845 & & \\
\hline \multirow{3}{*}{2} & \multirow{3}{*}{ Drug therapy } & Proprietary chinese medicines & $7.187^{\mathrm{a}}$ & 6.661 & 7.714 & \multirow{3}{*}{$P<0.01$} & \\
\hline & & herbs & $5.662^{\mathrm{a}}$ & 5.136 & 6.189 & & \\
\hline & & Western & $5.125^{\mathrm{a}}$ & 4.598 & 5.652 & & \\
\hline
\end{tabular}

Table 3: Tendency of TCM syndrome Differentiation and treatment.

\begin{tabular}{|c|c|c|c|c|c|}
\hline \multirow{2}{*}{ Serial Number } & \multirow{2}{*}{ Items } & \multirow{2}{*}{ Average } & \multicolumn{2}{|c|}{ 95\% Confidence Interval } & \multirow{2}{*}{$P$} \\
\hline & & & Lower & Ceiling & \\
\hline 1 & Wind chill Headache: Chuanxiong Chatiao San & 5.200 & - & - & \\
\hline 2 & Wind Fever headache: Xiongzhi shigao Decoction & 5.288 & - & - & \\
\hline 3 & Rheumatism Headache: Qianghuo Shengshi Decoction & 5.788 & - & - & \\
\hline 4 & Hyperactivity of liver Yang: Gastrodia and Uncaria Beverage & 5.900 & - & - & \\
\hline 5 & Phlegm-turbidity disturbing: Banxia Baizhu Tianma Decoction & 6.050 & - & - & \\
\hline \multirow{2}{*}{6} & Blood Stasis Syndrome: Tongqiao Huoxue Decoction & $5.725^{\mathrm{a}}$ & 5.208 & 6.242 & \multirow{2}{*}{$P>0.01$} \\
\hline & Blood Stasis Syndrome: Xuefu Zhuyu Decoction & $5.462^{\mathrm{a}}$ & 4.945 & 5.980 & \\
\hline 7 & Deficiency of Qi and Blood: Si-Wu-Tang & 6.300 & - & - & \\
\hline 8 & Yin Deficiency of Liver and Kidney: Yuan and Sleep & 5.313 & - & - & \\
\hline 9 & Liver Depression Symptom: Hemilateral Headache Decoction & 5.625 & - & - & \\
\hline
\end{tabular}

\section{Primary Headache}

Migraine: After eliminating the influence factors by covariance analysis, such as gender, age and educational level, the results shows that there are some differences in the tendency of patients to different interventions $(\mathrm{P}<0.01)$ in the treatment of migraine, as shown in Table 4. 2.5.2 Tension -Type Headache After using covariance to control the influence of gender, age and educational level factors, the results shows that there are differences in the tendency of patients to different intervention measures in the treatment of tension-type headache $(\mathrm{P}<0.01)$, as shown in Table 5. 
Table 4: Tendency of migraine intervention measures.

\begin{tabular}{|c|c|c|c|c|}
\hline \multirow{2}{*}{ Serial Number } & \multirow{2}{*}{ Items } & \multirow{2}{*}{ Average } & \multicolumn{2}{|c|}{$\mathbf{9 5 \% \text { Confidence Interval }}$} \\
\cline { 3 - 5 } & & & Lower & Ceiling \\
\hline 1 & Acupuncture & $5.875^{\mathrm{a}}$ & 5.346 & 6.404 \\
\hline 2 & Collateral-puncture-bleeding & $5.875^{\mathrm{a}}$ & 5.346 & 6.404 \\
\hline 3 & Yangxue Qingnao Granula & $5.287^{\mathrm{a}}$ & 4.759 & 5.816 \\
\hline 4 & Chuanxiong Chatiao San & $5.275^{\mathrm{a}}$ & 4.746 & 5.804 \\
\hline 5 & Xuefu Zhuyu Decoction & $5.150^{\mathrm{a}}$ & 4.621 & 5.679 \\
\hline 6 & Toutongning & $4.962^{\mathrm{a}}$ & 4.434 & 5.491 \\
\hline 7 & Duliang Capsule & $4.825^{\mathrm{a}}$ & 4.296 & 5.354 \\
\hline
\end{tabular}

Table 5: Tendency of stress-type headache intervention.

\begin{tabular}{|c|c|c|c|c|}
\hline \multirow{2}{*}{ Serial Number } & \multirow{2}{*}{ Items } & \multirow{2}{*}{ Average } & \multicolumn{2}{|c|}{ 95\% Confidence Interval } \\
\cline { 3 - 5 } & & & Lower & Ceiling \\
\hline 1 & Massage & $6.462^{\mathrm{a}}$ & 5.919 & 7.006 \\
\hline 2 & Acupuncture & $5.737^{\mathrm{a}}$ & 5.194 & 6.281 \\
\hline 3 & Yangxue Qingnao Granula & $5.262^{\mathrm{a}}$ & 4.719 & 5.806 \\
\hline 4 & Toutongning & $5.050^{\mathrm{a}}$ & 4.506 & 5.594 \\
\hline 5 & Acupoint Injection & $4.813^{\mathrm{a}}$ & 4.269 & 5.356 \\
\hline
\end{tabular}

Cluster Headache: After eliminating the influence factors by covariance analysis, such as gender, age and educational level, the results show that there is no significant difference in the tendency

Table 6: Tendency of cluster headache intervention.

\begin{tabular}{|c|c|c|c|c|c|}
\hline \multirow{2}{*}{ Serial Number } & \multirow{2}{*}{ Items } & \multirow{2}{*}{ Average } & \multicolumn{2}{|c|}{ 95\% Confidence Interval } & \multirow{2}{*}{$P$} \\
\hline & & & Lower & Ceiling & \\
\hline 1 & Acupuncture & $5.800^{\mathrm{a}}$ & 5.240 & 6.360 & \multirow{3}{*}{$P>0.01$} \\
\hline 2 & Gastrodin Injection & $4.925^{\mathrm{a}}$ & 4.365 & 5.485 & \\
\hline 3 & Toutongning & $4.850^{\mathrm{a}}$ & 4.290 & 5.410 & \\
\hline
\end{tabular}

\section{Conclusion}

The attention of patients to the outcome index is to prevent and reduce recurrence, less adverse reactions, cure, ease of treatment, rapid pain relief, whether it is within the scope of medical insurance, treatment costs. In the direction of the overall treatment measures to choose, they preferred non-drug therapy, which in turn tends to choose massage, acupuncture, other, drug therapy as a secondary choice, which in turn tends to choose proprietary Chinese medicine, Chinese herbal decoction, Western medicine. Patients have no special preference for TCM syndrome differentiation and treatment tendency of headache. In the treatment of migraine, patients tend to Acupuncture, Collateral-puncture-bleeding, Yangxue Qingnao Granula, Chuanxiong Chatiao San, Xuefu Zhuyu Decoction, Toutongning, Duliang Capsule, Tianshu Capsule. In order to treat tension-type headache, patients tend to massage, acupuncture, Yangxue Qingnao Granula, Toutongning, Acupoint Injection. There was no significant difference in the tendency of different intervention measures in the treatment of cluster headache. of patients to different intervention measures in the treatment of cluster headache $(\mathrm{P}>0.01)$, as shown in Table 6 .

\section{Discussion}

\section{Analysis of The Reasons for Patients' Preference and Values}

Some scholars define patient preferences and values as follow: The patient's view of health and life, priorities, beliefs, expectations, values, and goals, as well as the process by which individuals consider the potential benefits, hazards, costs, and inconveniences of one treatment plan compared to another [4]. Headache patients ' preference and values the results showed that patients are highly concerned about the therapeutic effect of headache, which may be related to the high incidence of headache [5] and great harm [6]. When there are two or more effective treatment options for different types of hazards and benefits, patients attach more importance to harm than to the benefits [7]. Therefore, patients pay close attention to the adverse reactions of treatment, and generally prefer to choose non-drug therapies such as massage or acupuncture, which are non-invasive and have little side effects. Patients are not concerned 
about the cost of treatment for headaches, which may be related to the low medical costs required to treat headaches.

\section{Impact of Patient Preferences and Values on The Devel- opment of Clinical Practice Guidelines for Headaches}

Evidence-based medicine is a medical practice process that follows evidence, emphasizing that patients ' preferences and values must be fully taken into account in medical decision-making
[8]. Clinical Diagnosis And Treatment Guide for TCM Internal Medicine (Headache) 2017 follows the latest evidence-based guidelines to develop basic principles, combining evidence-based evidence, expert consensus opinions, and patient preferences and values to form final recommendations.In terms of migraine and tension-type headaches, even though the quality of the evidence is not high, recommendations referring to the patient's preferences and value are made , as shown in Table 7.

Table 7: Tecommendations for the guidelines on migraines and tension-type headaches.

\begin{tabular}{|c|c|c|c|c|c|}
\hline Serial Number & Type of Headache & Therapeutic Measures & Quality of Evidence & Tendency & Recommendations \\
\hline \multirow{2}{*}{1} & \multirow{2}{*}{ Migraine } & Acupuncture & Low & High & Strong recommendation \\
\cline { 3 - 6 } & & Toutongning & Low & Low & Weak recommendation \\
\hline \multirow{3}{*}{2} & \multirow{3}{*}{ Tension headache } & Massage & Medium & High & Strong recommendation \\
\cline { 3 - 6 } & & Acupuncture & Medium & High & Strong recommendation \\
\cline { 3 - 6 } & & Toutongning & Low & Low & Weak recommendation \\
\hline
\end{tabular}

\section{Prospects and Shortcomings}

At present, the development of clinical guidelines in China rarely take the preferences and values of patients into account, which seriously affects the compliance of patients receiving treatment and the effectiveness of clinical treatment [9]. Most of the preferences and values of medical staff and patients tend to be the same, but sometimes there are differences [10]. As is shown in the survey, patients 'tendency to treatment cost is much lower than the expected results of medical investigators. As patients who experience the pain and treatment of diseases, they often have more to say in the choice of disease-related diagnosis and treatment. Clinical Diagnosis and Treatment Guide for TCM Internal Medicine (Headache) 2017 as the first clinical Practice guide of TCM combined with patients' preferences and values, the final recommendation of its formation is of great significance to the improvement of clinical curative effect.

In addition, we can also gradually try to carry out doctorpatient joint decision-making, doctor-patient common cases and other forms to improve patients' participation in the process of disease diagnosis and treatment, so as to further improve patients' compliance and the effectiveness of clinical treatment. There are still shortcomings in this study:

a) Use cross-sectional network survey methods, lacking randomized controlled objective rigor

b) The inclusion of survey subjects lack of strict diagnostic criteria and exclusion criteria c) The number of cases in which patients are collected to be investigated can be wider.

\section{References}

1. Sackett D, Straus S, Richardson W (2000) Evidence-based medicine. How to practice and teach EBM 2 ed. Edinburgh: Churchill Livingstone, UK.

2. (2011) IOM (Institute of Medicine). Clinical Practice Guidelines We Can Trust. The National Academies Press, Washington, DC, USA.

3. Yang Ke hu (2014) WHO Handbook for Guideline Development. Lanzhou University Press, Lanzhou, China.

4. Guyatt G H, Rennie D, Maureen O Meade, Deborah J Cook (2001) Users guide to the medical literature: essentials of evidence-based clinical practice. American Medical Association, p. 18-21.

5. Stovner L, Hagen K, Jensen R, Katsarava Z, Lipton R, et al. (2007) The global burden of headache: A documentation of headache prevalence and disability worldwide. Cephalalgia: an international journal of headache 27(3): 193-210.

6. Yu S, Liu R, Zhao G, Xiaosu Yang, Xiangyang Qiao, et al. (2012) The prevalence and burden of primary headaches in china: A populationbased door-to-door survey. Headache 52(4): 582-591.

7. Bergus GR, Levin IP, Elstein AS (2002) Presenting risks and benefits to patients. J Gen Intern Med 17(8): 612-617.

8. Strauss SE (2005) Evidence-Based Medicine: how to practice and teach Churchill Livingstone, UK, pp. 819-825.

9. Adamson SJ, Bland JM, Hay EM (2008) Patients'preferences within randomised trials: systematic review and patient level meta-analysis. BMJ 337: a1864.

10. Xie Ran, Chen Yao-long, Chen Hao, Wang Qi, Zhai Suo-di, Zhao Rongsheng, et al. (2015) Research methods of patients'values and preferences in the development of evidence-based guidelines. Chinese Journal of Evidence-based Mdeicine 15(05): 586-591. 


\section{ISSN: 2574-1241}

DOI: 10.26717/BJSTR.2019.15.002774

Hui Li. Biomed J Sci \& Tech Res

(C) (P) This work is licensed under Creative

Submission Link: https://biomedres.us/submit-manuscript.php

$\begin{array}{ll}\text { BIOMEDICAL } & \text { Assets of Publishing with us } \\ \text { RESEARCHES } & \text { - Global archiving of articles } \\ \text { - Immediate, unrestricted online access } & \text { - Rigorous Peer Review Process } \\ & \text { - Authors Retain Copyrights } \\ & \end{array}$

